\title{
Surface Plasmon Microscopy Measurements of Lipid Vesicle Adsorption on a Micropatterned Self-Assembled Monolayer
}

\author{
A. Toby A. J enkins,* Thomas Neumann, and Andreas Offenhäusser \\ Max-Planck-Institut für Polymerforschung, Ackermannweg 10, Mainz 55128, Germany
}

Received December 23, 1999. In Final Form: May 18, 2000

\begin{abstract}
This paper presents a novel method of measuring the adsorption of egg-phosphatidylcholine (egg-PC) vesicles onto a micropatterned heterogeneous self-assembled monolayer (SAM). Microcontact printing $(\mu \mathrm{CP})$ was used to make an array of mercaptoethanol patches surrounded by an octadecanethiol (ODT) SAM. Surface plasmon microscopy (SPM) followed the change in the different plasmon minima of the mercaptoethanol and ODT SAMs with respect to lipid adsorption time. Gray scale analysis (GSA) of the two domains showed clear differences in the kinetics of lipid adsorption, which were equated to a very different mechanism of lipid vesiclefusion on microcontact printed ( $u$ CP) SAMs compared with homogeneous SAMS. Surface plasmon microscopy combined with GSA has allowed us to spatially deconvolute the adsorption of lipid on a patterned surface, giving a unique insight into the lipid adsorption process. Conventional surface plasmon resonance measurements were made on the homogeneous SAM moieties in order to quantify the gray scale analysis in terms of adsorbed lipid thickness.
\end{abstract}

\section{Introduction}

The theory and background of surface plasmon microscopy (SPM) has been well documented by K noll et al. ${ }^{1}$ In simple terms it can be understood by considering how the angular position of the plasmon resonance minimum (created by the interaction of incident laser light with a thin metal film) is mediated by thethickness and refractive index of material on or very close to the metal. If a gold film is patterned with two self-assembling moieties of different thicknesses and/or refractive indices, one of the SAMs will come into resonance at a lower angle than the other. ${ }^{1}$ In conventional surface plasmon resonance spectroscopy (SPR) it is not possible to deconvolute the two minima and a spatially averaged spectra is obtained over the diameter of the spot of laser light. Surface plasmon microscopy utilizes a spatial filter to expand the laser beam in order to illumi nate the whole sample area. The reflected light is then detected by a CCD camera via a microscope lens. Providing the features on the surface arenot toosmall ( $>4 \mu \mathrm{m})$, thedifferent adsorption minima of the two SAM s can be seen. Previously SPM has been used to study biotin-streptavidin binding and antigenantibody binding on surfaces. ${ }^{2,3} \mathrm{~A}$ general discussion of SPM for analytical purposes is given by Yeatman. ${ }^{4}$

The adsorption of lipid vesicles on surfaces is an area of great current interest, since it is claimed that it is possible to create a biomimetic membrane on a surface which shares the properties of natural cytoplasmic membranes in terms of the functioning of incorporated proteins and peptides. ${ }^{5}$ These solid-supported lipid bilayers in principleallow transmembraneprotein function to bestudied and may form the basis of thenext generation of biosensors. Many of the solid-supported membrane

* Corresponding author. E-mail: a.t.a.jenkins@bath.ac.uk. Current address: Department of Chemistry, U niversity of Bath, Bath BA2 7AY, England.

(1) Rothenhäusler, B.; Knoll, W. Nature 1988, 332, 615-617.

(2) Häussling, L.; Ringsdorf, H.; Schmitt, F.J .; Knoll, W. Langmuir 1991, 7, 1837-1840.

(3) Fischer, B.; Heyn, S. P.; Egger, M.; Gaub, H. E. Langmuir 1993, 9, $136-140$

(4) Yeatman, E. M. Biosens. Bioelectron. 1996, 11, 635-649.

(5) Sackmann, E. Science 1996, 271, 43-48. systems documented in recent literature are dependent on the adsorption and self-organization of lipid vesicles on surfaces. ${ }^{6-11}$ Two recent studies have shown that it is possible to adsorb lipid vesicles onto a heterogeneous micropatterned surfaceand retain thefunction of peptides incorporated into themembrane, namely grami cidin and valinomycin. ${ }^{6,7}$ However, understanding the adsorption of lipid onto such micropatterned SAMs is far fromsimple, since theroles of threeseparate components of thesurface (thetwoSAM s and their interface) need to be consider ed. ${ }^{8}$ Studies by surfaceplasmon resonance(SPR) of li pid vesicle adsorption on hydrophobic SAMs point to the formation of a monolayer of lipid mol ecules. This can be understood by considering the surface energy advantages of making a hydrophobic surface hydrophilic in an aqueous medium. ${ }^{10}$ The system on a hydrophilic SAM is rather more complex, with various studies suggesting at least the partadsorption of lipid vesicles as well as a lipid bilayer. ${ }^{12}$

This paper describes the adsorption of egg-phosphatidylcholine (egg-PC) vesicles on a microcontact printed $(\mu \mathrm{CP})$ gold substrate consisting of an array of mercaptoethanol patches, $24 \mu \mathrm{m}$ in diameter with a center-center distance of $40 \mu \mathrm{m}$ surrounded by an octadecanethiol (ODT) SAM.

\section{Experimental Section}

Microcontact printingand production of patterned stamps have been extensively described by Whitesides in previous reports. ${ }^{13}$ Patterned stamps of poly(dimethyl sil oxane) (PDMS) weremade

(6) J enkins, A. T. A.; Bushby, R.J .; Boden, N.; Evans, S. D.; Knowles, P. F.; Miles, R. E.; Ogier, S. D. Langmuir 1998, 14, 4675-4678.

(7) J enkins, A. T. A.; Bushby, R.J .; Boden, N.; Evans, S. D.; Knowles, P. F.; Miles, R. E.; Ogier, S. D.; Schönherr, H.; Vancso, G. J . J . Am. Chem. Soc. 1999, 121, 5274.

(8) Evans, S. D.; J enkins, A. T. A.; Ogier, S. D.; Bushby, R. J . J . Electroanal. Chem. Submitted November 2000.

(9) Cornell, B. A.; Braach-Maksvytis, V. L. B.; King, L. G.; Osman, P. D. J .; Raguse, B.; Wieczorek, L.; Pace, R. J . Nature 1997, 387, 580583.

(10) Lingler, S.; Rubinstein, I.; Knol I, W.; Offenhäusser, A. Langmuir, 1997, 13, 7085-7091.

(11) Plant, A. L. Langmuir 1999, 15, 5128-5135.

(12) Rädler, J.; Strey, H.; Sackmann, E. Langmuir 1995, 11, 45394538.

(13) Xia, Y.; Whitesides, G. M. Angew. Chem., Int. Ed. 1998, 37, $4000-4025$. 


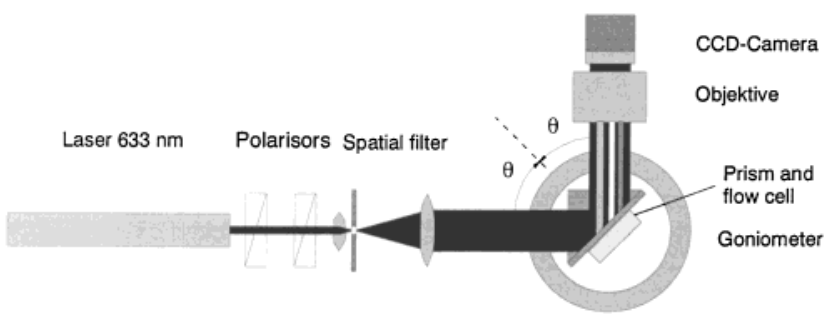

Figure 1. Schematic view of experimental surface plasmon microscopy setup.

by pouring liquid PDMS onto a piece of patterned photoresist on silicon, baked to cross-link the PDMS and peeled off, revealing a pattern of wells in thePDMS of the dimensions described above. The PDMS stamp was "inked" with $5 \mathrm{mM}$ ODT in HPLC grade ethanol, dried under nitrogen, and applied to a gold film. The gold film was of thickness $50 \mathrm{~nm}$, created by thermal evaporation of gold onto high refractive index LaSFN9 glass. The ODT patterned gold was subsequently "backfilled" with mercaptoethanol by immersion for 5 min in $5 \mathrm{mM}$ mercaptoethanol solution in ethanol. The patterned substrate was removed, rinsed in copious ethanol and water, dried under a stream of nitrogen, and placed onto an LaSF N $90^{\circ}$ prism, using index match fluid to ensureoptical continuity. A Teflon flow cell was applied to the SAM sideof thegold, and the prism-substrate-flow cell was placed in a surface plasmon microscope. ${ }^{8}$ Laser light from a He Nelaser at $633 \mathrm{~nm}$ wavelength was passed through a spatial filter and lens to create a broad coherent band of light which was directed onto the back of the micropatterned substrate via the prism (Kretschmann configuration), and ther eflected light was detected via a CCD camera. When the laser incident angle was stepwise increased by using a goni ometer, control softwareal lowed images to berecorded at defined angles. Theobtained SPM pictureseries shows the angl e dependent, laterally resolved reflectivity of the patterned surface. Software routines were then used to analyze the gray scale of defined areas in order to obtain SPM scan curves. During the kinetic measurements pictures were recorded in set time intervals at a fixed incident angle and were converted into numerical values by gray scaleanalysis (GSA). A schematic view of the experimental setup is shown in Figure 1.

Lipid vesides were made by hydration and subsequent extrusion (through $50 \mathrm{~nm}$ diameter pores) of freshly hydrated egg-PC prior to dilution to $0.2 \mathrm{mg} / \mathrm{mL}$ in citrate-phosphatebuffer at $\mathrm{pH} 5.5 .^{6}$

Conventional SPR measurements were made of lipid adsorption on homogeneous liquid formed SAMs of both the mercaptoethanol and the octadecanethiol (ODT). The plasmon curves thus obtained werefitted using theF resnel equations, to a model layer systemall owing the calculation of thethickness of adsorbed lipid on the SAMs (assuming a lipid refractive index of 1.5).

\section{Results and Discussion}

SPR of Lipid Adsorption on Homogeneous SAMs. Measurements of lipid adsorption on the homogeneous SAMs are given in Figure 4. The thickness found for the lipid on the ODT SAM was $18 \AA$, consistent with a lipid monolayer being formed. H owever, measurements on the mercaptoethanol SAM gave a final thickness of $>80 \AA$, suggesting either lipid multilayers or vesicle adsorption. Thethickness of lipid on theODT layer was used to convert the gray scale data to thickness. The gray scale value at $\mathrm{t}=0$ was set to $0 \AA$ adsorbed lipid, on both the ODT and mercaptoethanol areas. The change in gray scale from $t$ $=0$ to after vesicle adsorption and rinsing on the $\mu \mathrm{CP}$ ODT was equated to a thickness of $18 \AA$. Hence, it was possible to calibrate the gray scale in terms of film thickness, so allowing the thickness of lipid adsorbed in the mercaptoethanol areas to be calculated. These measurements assume that the $\mu \mathrm{CP}$ ODT and the liquid formed ODT have similar properties. This seems not unreasonable, given that high-resolution AFM images of the $\mu \mathrm{CP}$ ODT show a clear hexagonal lattice of ODT

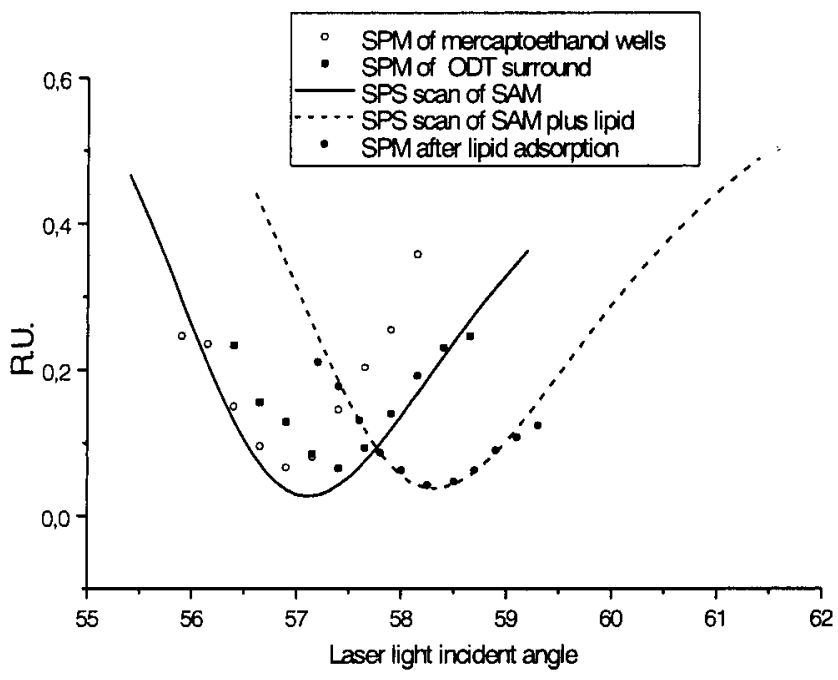

Figure 2. SPR adsorption curves around the reflectance minimum, measured by conventional SPR (before and after lipid adsorption) and SPM with GSA.

molecules (on terraces of $A u(111)$ ), previously seen on liquid formed ODT SAMs (not shown here). These measurements areconsistent with work doneby Libioulle et al., which suggests that the qual ity of $\mu \mathrm{CP}$ SAMs can be made closer to that of liquid formed SAMs by using a higher concentration of "inking" thiol solution. ${ }^{14}$

SPM and SPR of Lipid Adsorption on Patterned SAMs. A conventional SPR curve of the patterned substrate was obtained prior to conversion of the instrument to a surface plasmon mi croscope. This was done to find the averagesurfaceplasmon minima of thesubstrate beforetheadsorption of thevesicles. Subsequently, a SPM scan was recorded of the patterned substrateas described above. In the gray scal eanalysis, 10 separatepoints (from each image) from the mercaptoethanol region and 10 separatepoints from the ODT SAM region wereanalyzed. The gray scale of these points was averaged to give the plasmon minima curves seen in Figure 2.

As expected, thethinner mercaptoethanol patches come intoresonance first, indicated al so by a contrast inversion when the incident angle of the laser light was changed around the averaged plasmon mi nimum position. As can beseen from $\mathrm{F}$ igure 2, the mean value of the deconvoluted plasmon minima positions coincides with the minimum of the averaged SPR curve before vesicle adsorption.

For the kinetic measurements a fixed angle was then chosen of slightly lower angle $\left(56.4^{\circ}\right)$ than the plasmon minima, at which the greatest contrast in resonance between the ODT and mercaptoethanol could be seen. At this position the slope of the single-scan curves is large enough to make the kinetic measurements sensitive to very small shifts of the plasmon minima during adsorption of molecules to the SAMs. Figure 3 shows single images from the recorded series taken at times that correspond to markings in Figure 4. The SPM image of the substrate at the chosen incident angle of $56.4^{\circ}$ at time $t=0 \mathrm{~s}$ is shown in Figure 3A. Thearray of darker mercaptoethanol patches (in resonancefirst) can cl early beseen surrounded by a lighter ODT SAM.

On injection of lipid vesicles to the flow cell, the image changed rapidly. Within $300 \mathrm{~s}$ it was possible to discern that the image had almost inverted. In image 3B, at 800 $\mathrm{s}$, it can clearly be seen that the array of dark hydrophilic

(14) Libioulle, L.; Bietsch, A.; Schmid, H.; Michel, B.; Delamarche, E. Langmuir 1999, 15, 300-304. 


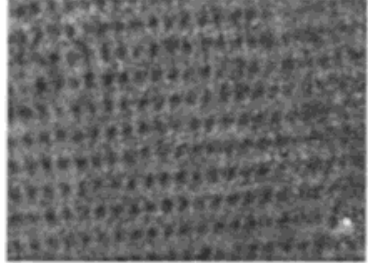

A (0 s)

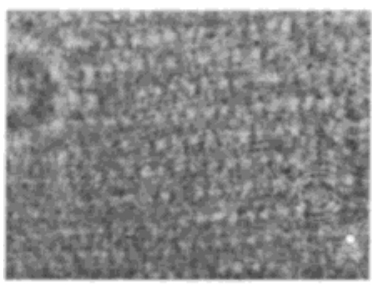

$\mathrm{C}(4000 \mathrm{~s})$

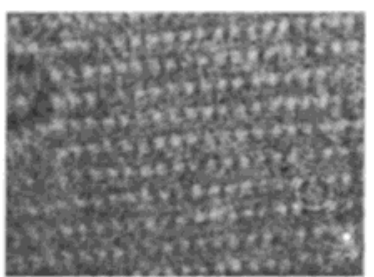

B (300 s)

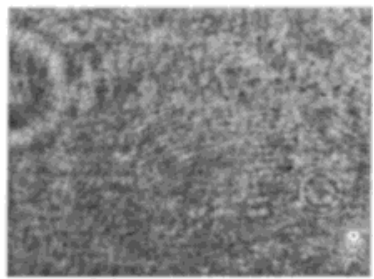

$\mathrm{D}(25000 \mathrm{~s})$
Figure 3. SPM pictures taken during the kinetic experiment at times $A-D$.

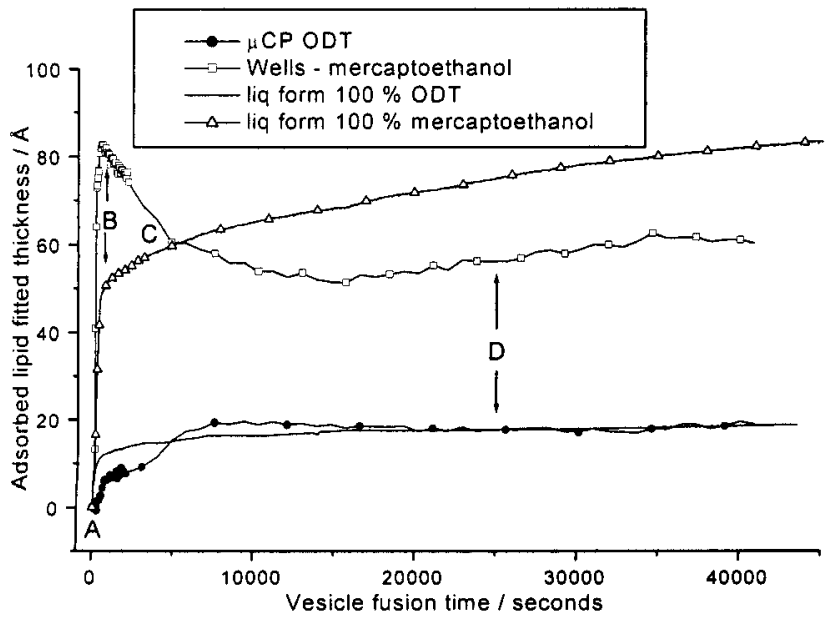

Figure 4. Kinetics of lipid adsorption on hydrophilic mercaptoethanol wells and thesurrounding hydrophobic ODT and, for comparison, on nonpatterned homogeneous liquid formed SAMs of the two thiol moieties.

patches is now brighter than the surrounding area, indicative that a greater thickness of material is present in this region than on the surrounding ODT SAM. By $4000 \mathrm{~s}$ into the vesicle fusion, the contrast starts to fade 3C, until, by image 3D at $25000 \mathrm{~s}$, the image appears virtually homogeneous. A total of 165 images were recorded over thetime of the kinetic experiment and were analyzed by GSA in order to obtain the kinetics of the vesicle adsorption to the two different thiol patches. The gray scale of the same number of points as for the scan curves has been averaged and converted to thickness values (vida supra), as shown in Figure 4. A rinsing step was also carried out to determi neany change of thickness after removing loosely adhered material. Within the resolution possible by SPM, no quantifiable change was seen.
After the kinetic measurement was stopped, further SPR and SPM scans of the surface were recorded to evaluate the thickness change due to the adsorption process (Figure 2). Note, because the image after lipid adsorption was homogeneous (Figure 3D), it was not possible to deconvolute the plasmon minima of the mercaptoethanol and ODT areas. Therefore, theSPR and SPM scan curves after theadsorption areidentical (Figure 3).

Adsorption on ODT SAM. Although the adsorption curve is inevitably noisier than that obtained by conventional SPR, the basic shape of the adsorption curve fits very well to previous measurements we and others have made of egg-P C lipi d adsorption on 100\% ODT SAMs. $8,10,11$

Adsorption on Mercaptoethanol Patches. TheGSA analysis of lipid adsorption on the mercaptoethanol wells has proved to be rather interesting, showing a marked difference in the adsorption measured by conventional SPR on $100 \%$ mercaptoethanol SAMs wherea sharp initial adsorption is seen followed by a long period of gradual increase in reflectance/thicknes but no adsorption peak followed by a decline in reflectance was seen (F igure 4). This suggests that adsorption of egg-PC vesicles in the mercaptoethanol patches on the patterned SAM is affected strongly by the edges of the patches, that is to say, the hydrophilic/hydrophobic SAM interface. A reasonable explanation could bethat vesicles in the mercaptoethanol patches initially adsorb as vesicles and sit on the surface. A much longer process then takes place in which the vesicles fuse with each other (Figure 4) and collapse on contact with the surrounding hydrophobic area to form a lipid bilayer. The end result of such a period of vesicle collapse is seen in Figure 3D, where the surface appears uniform although measurement of the total adsorbed thickness of the lipid on the $\mu \mathrm{CP}$ SAM by conventional SPR suggests that there is still significantly more lipid adsorbed than would be expected for a simple lipid mono/ bilayer. Such an explanation would be supported be a study previously carried out on similar systems, wherea range of mercaptoethanol areas were studied using conventional SPR and AFM.8

\section{Conclusions}

The results presented in this paper show that lipid adsorption onto heterogeneous patterned SAMs is a far morecomplex process than previously thought. Thespatial deconvolution of the surface plasmon resonance has allowed a remarkableinsight intothe possiblemechanism of lipid adsorption in the mercaptoethanol patches on the patterned SAM, which shows theprocess is quitedifferent than that seen on homogeneous mercaptoethanol SAMs. The contrast inversion seen in Figures 3 and 4 is most interesting.

Acknowledgment. A.T.A.J . would like to acknowledgethefinancial support of theAlexander-von-Humbol dt Stiftung for this work and interesting discussions with S. D. Evans (University of Leeds, U.K.).

\section{LA9916800}

